\title{
Me first? Positioning self in the attentional hierarchy
}

Article

Accepted Version

Cunningham, S., Vogt, J. ORCID: https://orcid.org/0000-00023178-2805 and Martin, D. (2022) Me first? Positioning self in the attentional hierarchy. Journal of Experimental Psychology: Human Perception and Performance, 48 (2). pp. 115-127. ISSN 0096-1523 doi: https://doi.org/10.1037/xhp0000976 Available at https://centaur.reading.ac.uk/100880/

It is advisable to refer to the publisher's version if you intend to cite from the work. See Guidance on citing.

To link to this article DOI: http://dx.doi.org/10.1037/xhp0000976

Publisher: American Psychological Association

All outputs in CentAUR are protected by Intellectual Property Rights law, including copyright law. Copyright and IPR is retained by the creators or other copyright holders. Terms and conditions for use of this material are defined in the End User Agreement.

\section{www.reading.ac.uk/centaur}

\section{CentAUR}

Central Archive at the University of Reading 
Reading's research outputs online 


\title{
Me first? Positioning self in the attentional hierarchy
}

\author{
Sheila J Cunningham ${ }^{\mathrm{a}}$, Julia Vogt ${ }^{\mathrm{b}}$, and Douglas Martin ${ }^{\mathrm{c}}$
}

\author{
Affiliations \\ a. Division of Psychology, Abertay University, Dundee, UK, DD1 1HG \\ b. School of Psychology and Clinical Language Sciences, University of Reading, Reading, \\ Berkshire, UK, RG6 6AH \\ c. School of Psychology, University of Aberdeen, King's College, Aberdeen, UK, AB24 3FX
}

WORD COUNT: 9,826

Corresponding author: Prof Sheila Cunningham: s.cunningham@abertay.ac.uk

Other author contacts: Dr Doug Martin: doug.martin@abdn.ac.uk

Dr Julia Vogt: j.vogt@reading.ac.uk 


\begin{abstract}
The attention system that allocates resources across competing aspects of the environment is influenced by biases toward particular types of stimuli, such as cues of threat (e.g., angry-face image), self-reference (e.g., own-face image) and current goals (e.g., food image when hungry). Here, we used dot probe tasks to investigate which of these stimulus types are prioritized in the attentional hierarchy, measuring response latency to dot probes presented in the same location as different face types. In Experiment 1, participants $(N=42)$ were presented with self, angry and neutral face images in the dot probe task, which revealed a clear attentional bias for self-images over both angry and neutral images. In Experiment 2, each participant $(N=69)$ was assigned a self, angry or neutral goal image for a secondary monitoring task designed to induce a temporary goal, and this image was included in the stimuli presented in the dot probe task. Again, self-cues were found to produce a strong attentional bias, but images associated with temporary goals were found to be the most effective source of attentional bias. Results are discussed in relation to the relative importance of self, threat and temporary goal cues in the attentional hierarchy.
\end{abstract}

Keywords: attentional bias, self, threat, temporary goals, dot probe task

Public significance statement: The reported research shows that when different cues in the environment compete for attention, those most relevant to current goals are prioritized, followed by those that are relevant to self. Exploring this order of priority reveals the dynamic nature of attentional biases, which has relevance for the way in which cues are used to attract attention in the real world (e.g., to engage students in learning). While selfprioritization biases have already been applied in this way, the current findings suggest manipulating goal-relevance may be even more effective. 
One of the basic tenets of attention is that it has a limited capacity, such that it is not possible for us to attend simultaneously to multiple aspects of space (Desimone \& Duncan, 1995; Oberauer, 2019). The allocation of attention is therefore fundamentally important to shaping cognition (Posner \& Rothbart, 2007). Research has identified a number of classes of stimulus towards which attention is drawn, demonstrating attentional biases for specific aspects of the environment (e.g., threatening stimuli, emotional facial expressions, current goals, information associated with self; e.g. Humphreys \& Sui, 2016; Pourtois et al., 2013; Vogt et al., 2013; Yiend, 2010). These attention biases determine the information that is perceptually prioritized and subsequently processed (Everaert et al., 2012; Luck \& Vecera, 2002). An interesting question in attention allocation is what happens when multiple stimuli in the environment are potentially attention-biasing - which cues are dominant in an attentional hierarchy? The current study aimed to investigate this issue by examining the relative dominance of three types of stimuli known to bias attention - threat, self-cues and current goals.

The class of stimuli perhaps most widely associated with attention capture is threatening cues, with words and images associated with danger often shown to elicit attentional biases (for overviews see Vuilleumier \& Huang, 2009; Yiend, 2010). For example, when an angry face and a neutral face are presented together onscreen, stimuli subsequently presented in the same onscreen location as the angry face are responded to more quickly than those co-located with the neutral face (Mogg \& Bradley, 1999; see also Kuhn et al., 2015). Similarly, in visual search tasks angry faces are easier to pick out of an array than those with other expressions, and threatening cues like fear-conditioned stimuli are easier to find than neutral stimuli (e.g., Hodsoll et al., 2011; Notebaert et al., 2011). While the strength and reliability of these effects can vary (Schettino et al., 2013; Vuilleumier \& Huang, 2009), there are argued to be adaptive values of this system: biases to threatening cues ensure that 
potentially dangerous stimuli in the environment (e.g., attackers) are preferentially processed, facilitating faster responses that give the perceiver a better chance of survival (see Öhman \& Mineka, 2001; Vuilleumier \& Huang, 2009).

A similar attention-biasing effect to threat has been identified for self-relevance, with cues such as one's own name or face incurring preferential processing (Humphreys \& Sui, 2016). Like threat, this priority processing is argued to have an adaptive function, ensuring that information of potential importance to self is not missed (Cunningham, 2016). Any cues that are strongly associated with the self (e.g., one's own hometown or belongings) can trigger an attentional bias (Bargh, 1982) with own-faces and own-name cues, for example, being detected reliably more quickly than other images in visual search tasks (Harris \& Pashler, 2004; Tong \& Nakayama, 1999; Yang et al., 2013). Self-associated cues are detected earlier in the visual processing stream, and elicit additional visual discriminability (Sui et al., 2012; Macrae et al., 2017). Self cues also capture attention, such that their presentation can disrupt the detection and processing of co-presented stimuli (Bargh \& Pratto, 1986; Brédart et al., 2006; Röer et al., 2013; Yin et al., 2019; for a review see Humphreys \& Sui, 2016). Auditory as well as visual self-biases are robust, as demonstrated by the long-established 'own name effect', whereby hearing one's name in an unattended channel diverts attention away from current tasks (Moray, 1959; Wood \& Cowan, 1995). Interestingly, the bias to selfcues can be adapted to meet short-term task demands, with stimuli that are temporarily associated with self during a task (e.g., 'You are the triangle') incurring similar biases to those associated with self across the lifespan like one's own name (Sui et al., 2012; Yin et al., 2019). These effects suggest that any stimuli that are relevant to self have the capacity to bias attention.

An interesting empirical question that remains unanswered is the interaction between these effects: what happens when adaptive attentional biases like threat and self-relevance are 
in competition with one another? This question is important because examining the results of competition between attention-biasing cues will not only highlight how attention is allocated in real-life situations that contain these cues in competition, but also provides key theoretical insights. For example, research exploring the effects of competing endogenous to exogenous orienting cues has shaped our understanding of their mediating and independent effects (e.g., Berger et al., 2005; Grubb et al., 2015), and research on the competition between exogenous cues such as color, luminance and spatial distribution forms the foundation of our understanding of perceptual development (e.g., Dannemiller, 2000). Thus, exploring the results of competition between cues of self-relevance and survival would provide a deeper understanding of how these different long-term goals shape cognition.

Given the immediate existential jeopardy associated with threatening stimuli, it could be argued that these should always be prioritized over self-cues in the attention system. Indeed, some authors argue for a general attention bias for negative over positive stimuli (Ito et al., 1998; Öhman \& Mineka, 2001; Pratto \& John, 1991; although see Becker, Anderson et al., 2011; Vogt et al., 2020). Since threat cues are inherently negative while self is considered a positive stimulus (Beggan, 1991; Robins \& Beer, 2001), this would indicate that threatening cues should be positioned higher than self-cues in the attentional hierarchy. On the other hand, theorists have argued that self is 'special' in its role in driving perceptual prioritization (see Humphreys \& Sui, 2016). Conway and colleagues argue that self is a perpetually-active goal, continually primed by building an autobiographical memory system around selfreferenced representations and information consistent with the self-concept, as well as shortand long-term self-relevant goals (see Conway, 2005; Conway \& Pleydell-Pearce, 2000). Thus, an argument could also be made for self-cues being situated at the top of any hierarchy of attention allocation. Finally, it is possible that long term adaptive goals such as monitoring for threat and self-relevance may be moderated by short term goals (cf. Hahn \& Gronlund, 
2007). Research suggests that a top-down temporary goal such as monitoring for a specific content can actually over-ride longer-term survival goals (Victeur et al., in press; Vogt et al., 2013) so these may actually take prime position. As these theoretical arguments demonstrate, the prioritisation of different attention-biasing cues is far from resolved.

The current study is designed to explore these arguments. In Experiment 1, a dot probe task was used to compare attentional bias towards angry and self-relevant images, while in Experiment 2 this was combined with a temporary goal task to examine the interaction between angry and self-relevant cues that vary in in their relevance to temporary goal. Together, these experiments will provide new insights into prioritisation within the attention allocation hierarchy.

\section{Experiment 1: Self v. Angry images}

Experiment 1 was designed to directly compare the influence of threatening and selfrelevant cues for the first time, in the absence of temporary goals. Participants were asked to respond to dot probes presented after image pairs comprising combinations of self, angry and neutral faces. A dot probe task was used because it allows a direct comparison of the impact of the two images when competing against each other, either of which could attract attention. In this version of the paradigm neither of the images predict the probe location, so that the attention biasing effect of the stimulus categories can be determined in the absence of the potential confound of location task relevance. If attention is directed toward one image, it should facilitate responses on 'congruent' trials (i.e., when the subsequent probe presented in the same location) while increasing response time to 'incongruent' trials (i.e., when the probe is presented in the other location; see Macleod et al., 1986; Vogt et al., 2010). Here, a dot probe was located in the same position as one of two face images presented together, taken from a set of self images (own face), threatening images (unknown face with angry 
expression) and neutral images (unknown face with neutral expression). We used three trial types in this paradigm: self image versus neutral image, angry image versus neutral image, and angry image versus self image. This allowed us to test whether angry face and self cues bias attention when presented against a neutral category but also when presented against each other.

\section{Method}

\section{Participants and design}

Forty-two Abertay University students ( 35 female, $M_{\text {age }}=19.6$ years, $S D_{\text {age }}=3.5$ ) participated in the study. The effect associated with attention-capturing stimuli can vary across different experimental paradigms (see Bar-haim et al. 2007 for meta-analytic review) but we calculated a sample size based on the effect size of $d=0.55$ reported by a closelyrelated paradigm (threatening v. neutral stimuli comparison, Vogt et al., 2013), at an alpha of 0.05 and power of 0.80 . Using these figures, $G^{*}$ Power gave a required minimum sample size of 38. Vogt et al. used the following formula to calculate Cohen's $d_{z}:\left|\mathrm{M}_{1}-\mathrm{M}_{2}\right| / \mathrm{SQRT}\left(\left(\mathrm{SD}_{1}{ }^{2}\right.\right.$ $\left.+\mathrm{SD}_{1}{ }^{2-}\left(2 \mathrm{rSD}_{1} \mathrm{SD}_{2}\right)\right)$ which is recommended for repeated measures, within-subjects designs (Lakens et al., 2013; Faul et al., 2007). In this paper, we will report Cohen's $d$ calculated with the same formula for attentional bias effects. Stopping rule was completion of weekly participation sign ups once an appropriate sample size was reached. All participants had normal or corrected-to-normal vision and were naive as to the purpose of the experiment.

The experiment had a repeated-measures design of Face type (self, angry, neutral) and probe Congruence (congruent, incongruent), with the dependent variable being response latency to dot probes presented in the location each face type. Ethical approval for the experiment was provided by Abertay University’s Research Ethics Committee. 


\section{Apparatus and Materials}

The experiment was programmed using the EPrime 2.0 software package that was implemented on an HP Elite Desk PC a 19-inch LCD monitor. All stimuli were presented against a black background.

\section{Face images}

Three self-images were taken of each participant (a front, left- $3 / 4$ and right- $3 / 4$ face view) with a light blank background. The faces of four models from the Radboud Face Database were used as the non-self images (database IDs: 01, 02, 05 \& 07; Langner, et al., 2010; gender-matched to each participant and similar in age and ethnicity to the sample majority), also presented on a light blank background. All views of one model were presented with a neutral expression for each participant, with all views of the other model being angry. Assignment of model to face type (angry or neutral) was counter-balanced across participants. Each face image was 650 x 482 pixels in size and in full color.

\section{Dot probe task}

A trial in the dot probe task started with a fixation screen consisting of a white fixation cross (4 mm high) in the middle of the black screen (see Figure 1). Two white boxes $(10.7 \mathrm{~cm} \times 10.7 \mathrm{~cm})$ were presented left and right of the fixation cross, with the center of the boxes $11.0 \mathrm{~cm}$ from the fixation cross. After $500 \mathrm{~ms}$, a picture was presented simultaneously in each box for $350 \mathrm{~ms}$. Immediately after picture offset, a dot probe consisting of a small black square $(1.3 \mathrm{~cm} \times 1.3 \mathrm{~cm})$ appeared in the center of one of the boxes. Participants had to indicate the location of the target by pressing one of two keys ("S", "K") with left and right index finger on a QWERTY keyboard. A trial ended after a response was registered or 1500 ms had elapsed since probe onset. Correct responses elicited no feedback (a blank interval of 
$1000 \mathrm{~ms}$ duration) while erroneous and no responses elicited error feedback ('Incorrect' or 'Too slow!') for $1000 \mathrm{~ms}$. A 500ms blank interval preceded the start of the next trial.

Figure 1: Experiment 1 trial schematic

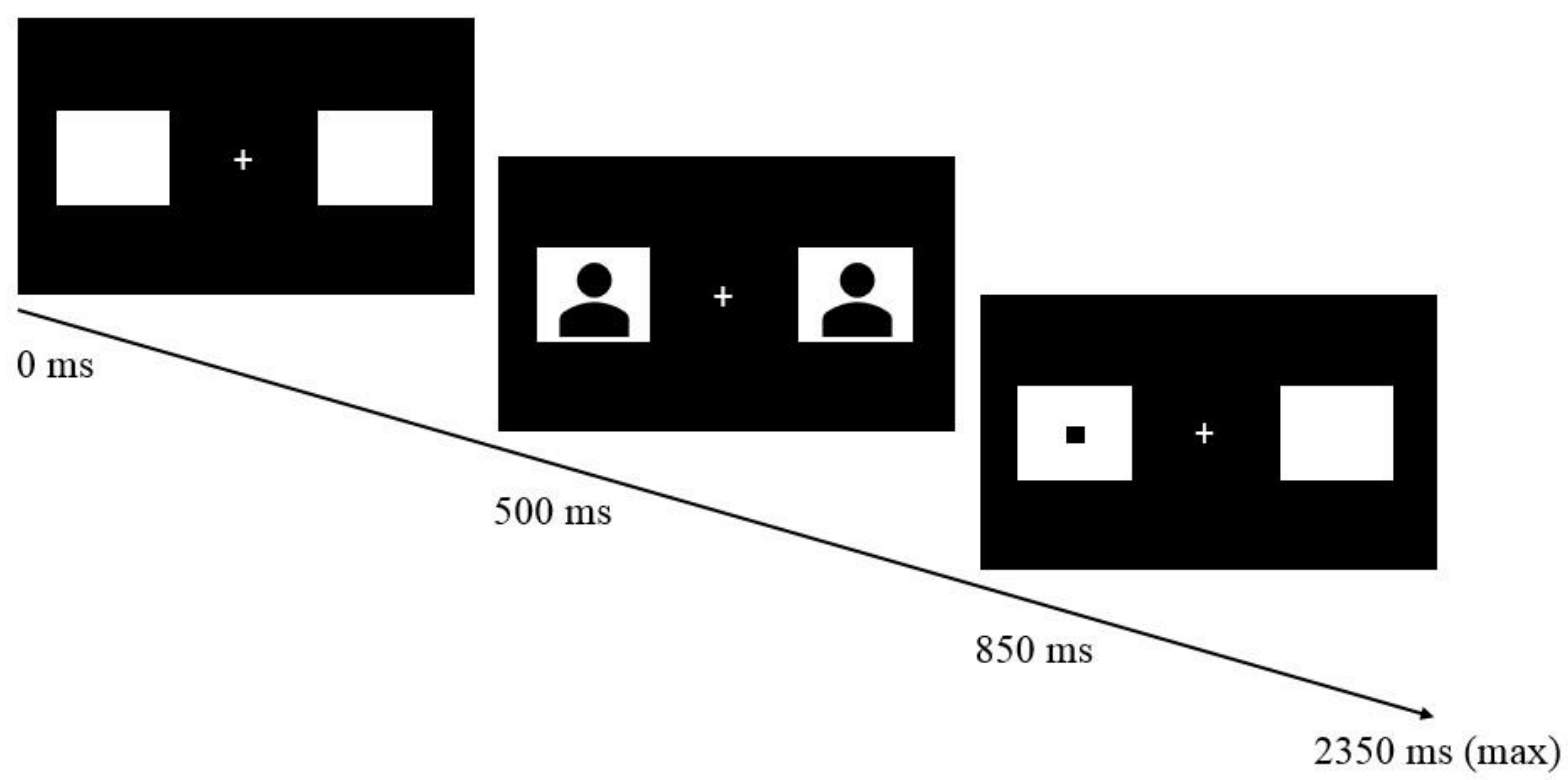

Interspersed in the task were nine 'digit trials' designed to maintain participants' fixation on the center of the screen (see Vogt et al., 2010). On these trials, the fixation cross was replaced by central presentation of a randomly selected digit ( $5 \mathrm{~mm}$ high) for $500 \mathrm{~ms}$, followed by the fixation cross for a further $10 \mathrm{~ms}$. Participants were instructed to report aloud the letter presented to ensure they were attending correctly to the screen; responses were not recorded but the experimenter alerted participants if any digits were missed or incorrectly reported. A $1000 \mathrm{~ms}$ blank interval preceded the start of the following trial.

\section{Procedure}

Participants were told they would be completing an image processing task and that one image would be their own face, requiring a digital photograph to be taken. After the participant's photograph was taken and uploaded to the programme, they were invited to sit 
at a table with the PC and keyboard, approximately 60 am away from the screen. Instructions were presented on the screen. The task began with 26 practice trials before participants were asked if they were ready to begin the test phase trials. The test phase consisted of $144 \mathrm{dot}$ probe trials and nine digit trials. We presented three different cue combinations in the attention trials: self vs. neutral control face, self vs. angry control face, and angry control face vs. neutral control face. Each combination was presented 48 times. Each image category was presented equally often in the left and right location and predicted the target location correctly on half of the trials. The order of trials was determined randomly and for each participant separately.

This study was not pre-registered but the full dataset can be accessed at https://osf.io/4re5b/.

\section{Results}

Trials with errors were removed (1.79\%). Following Vogt et al. (2013), reaction times (RTs) faster than $150 \mathrm{~ms}$ and slower than $1000 \mathrm{~ms}$ (1.21\%) were excluded, then mean RT per condition was calculated as the dependent variable. The data of one participant was excluded because they committed errors on more than $30 \%$ of the trials (more than the sample mean error \% plus three times the standard deviation of the sample's error \%).

To test the attentional bias toward self, angry and neutral pictures, RTs for 'congruent' images (i.e., target is presented in the same location as that face) were compared with 'incongruent' (i.e., target is presented in the other location) trials. For example, when face type is 'self', congruent trials were trials in which the target replaced the self-image, whereas incongruent trials were those in which the target replaced either the angry or neutral face. Paired comparisons examined the different effects of congruence across the three face types separately. These revealed reliable congruence effects in the Self face trials $[F(1,40)=$ 
$\left.13.95, p<.001, \eta_{\mathrm{p}}{ }^{2}=0.259, d z=0.61\right]$, with faster reactions on self-congruent trials $(M=$ $379 \mathrm{~ms} ; S D=70 \mathrm{~ms})$ than on self-incongruent (i.e., neutral or angry congruent) trials $(M=$ $391 \mathrm{~ms} ; S D=68 \mathrm{~ms})$. There was no congruence effect for neutral trials $[F(1,40)=1.72, p=$ 0.197. $\left.\eta_{\mathrm{p}}{ }^{2}=0.041, d z=0.19\right]$. However, interestingly there was a significant 'incongruence effect' in the angry face trials, with angry-congruent faces eliciting a slower RT $(M=390 \mathrm{~ms}$; $S D=72 \mathrm{~ms})$ than angry-incongruent (i.e., self-congruent or neutral-congruent) trials $(M=$ $383 \mathrm{~ms} ; S D=67 \mathrm{~ms})\left[F(1,40)=4.51, p=.040, \eta_{\mathrm{p}}^{2}=0.101, d z=0.35\right]$.

The incongruence effect in Angry face trials could be attributable to a self-bias, because $50 \%$ of the angry-incongruent trials were self-congruent. To explore this further, the effects of image congruence (i.e., whether the dot probe was congruent with one image vs. the other image in each pairing) were examined separately for the set of trials comprising each face type pairing (see Figure 2 for means within each pairing) A Self-congruent vs. Angry-congruent univariate ANOVA confirmed a significant self-congruence advantage in the Angry vs. Self trials $\left[F(1,40)=7.74, p=.008, \eta_{\mathrm{p}}{ }^{2}=0.162, d z=0.42\right]$, with the selfcongruent trials eliciting faster RTs than the angry-congruent trials. Similarly, a Selfcongruent vs. Neutral-congruent ANOVA showed a significant self-bias in the Self vs. Neutral trials $\left[F(1,40)=7.47, p=.009, \eta_{\mathrm{p}}{ }^{2}=0.157, d z=0.44\right]$. However, there was no corresponding difference in RT between angry-congruent and neutral-congruent trials in the Angry vs. Neutral pairings $\left[F(1,40)=0.15, p=.704, \eta_{\mathrm{p}}{ }^{2}=0.004, d z=0.04\right]$. 


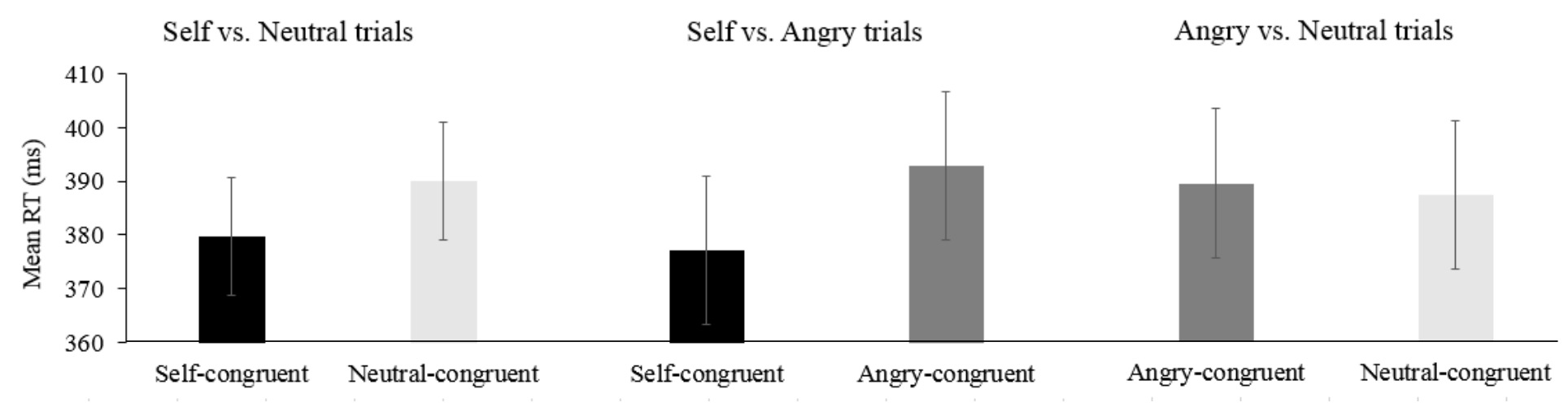

Figure 2: Mean response times by target congruence across all face-type pairings. (Error bars represent one standard error of the mean.)

The data reveal a consistent self but not angry bias, raising the possibility that the angry stimuli in the current experiment were not sufficiently salient to orient attention. This null finding is not unusual, as some previous studies report failures to find attentional bias to angry faces, especially in samples that are not selected to be clinically anxious (e.g., Schettino et al., 2013). However, secondary analysis was conducted so that the robustness of the attentional bias to self cues in the presence of angry faces could be ascertained. The analysis on Self vs. Angry trials was therefore repeated with a sub-sample of participants who displayed an attentional bias to angry faces on trials Angry vs. Neutral trials $(n=22$; face type effect $F(1$, 21) $\left.=48.94, p<.001, \eta_{\mathrm{p}}{ }^{2}=0.700, d z=1.4\right)$. Even in this smaller sample, a significant main effect of self-congruence on trials comparing self to angry face cues was found $[F(1,21)=$ $\left.5.08, p=.035, \eta_{\mathrm{p}}{ }^{2}=0.195, d z=0.50\right]$ revealing an attentional bias towards self cues with faster reactions on self-congruent trials $(M=364 \mathrm{~ms} ; S D=58 \mathrm{~ms})$, than on self-incongruent trials $(M$ $=375 \mathrm{~ms} ; S D=67 \mathrm{~ms}$ ).

\section{Discussion}


In Experiment 1, response latency in the dot probe task revealed that probes located in the same position as own-face images elicited faster response times than those located in the same position as another face image, regardless of whether that face was neutral or angry. Angry faces did not elicit an attention allocation effect when presented together with selffaces. A potential issue could be that the experiment was underpowered, given that a slightly larger sample size would be recommended based on the effect size for threat cues reported in Bar-Haim et al.'s (2007) cross-paradigm meta analysis $(d=0.45$, giving a suggested sample of 56 based on alpha of 0.05 and power of 0.80 ). However, even among participants who showed a bias for angry faces when paired with neutral faces in the current experiment, we found an attentional bias to self faces when they were paired with threatening faces, suggesting that power issues did not affect this pattern of results. Rather, these patterns suggest that self-cues are dominant relative to threatening cues in the attentional hierarchy. The attentional bias for self-cues is consistent with much previous research, which shows a reliable tendency for stimuli associated with self (e.g., own names, own faces, owned objects) to attract visuo-spatial and auditory attention (for review see Humphrey \& Sui, 2016). However, the novel finding that self-cues were more effective at directing attention than angry cues provides a new insight into their level of prioritisation.

The aim of Experiment 1 was to directly compare the attentional effects of self and angry cues, both of which are associated with long-term adaptive goals (Cunningham, 2016; Öhman, 2007). However, it is important to note that attention allocation is strongly influenced by short-term goals that also have the capacity to produce involuntary shifts in attention (Folk et al., 1992; Yantis, 2000), perhaps even over-riding long-term threat bias (Vromen et al., 2015). For example, Mogg et al. (1998) used a dot probe task to gauge attention allocation to food-related words and found that hungry participants displayed a stronger attentional bias to these food cues than participants who are not hungry (see also 
Tapper et al., 2010). Physical needs can thus induce short-term goals that guide attention, but research suggests that such goals can also be induced by attentional task demands. For instance, Folk et al. (1992) demonstrated attentional bias towards colored (but not uncolored) cues in participants who had a task goal of localizing a colored target stimulus, a bias that not apparent in participants who had a different target detection task. Task demands can therefore create temporary goals that guide cognitive processing.

To examine the influence on attention of active temporary goals induced in a concurrent task, Vogt et al. (2010) explored the impact of goal-relevant words on attentional processing during trials in which the goal was not task-relevant to the attention task. They designed a spatial cueing task in which participants were instructed to monitor for the presence of particular goal words for a reward in a secondary goal-inducing task that alternated with trials of the attention task. On alternate attention trials, participants completed a spatial cueing task in which words (sometimes including the goal word) were presented to the left or right of center, followed by a dot probe that was either congruent or incongruent with the word location. Response latency showed that congruence effects (i.e., facilitated response times on congruent trials) were significantly greater for goal words, even though the goal was not relevant in the spatial cueing trials. This suggests that temporary goals can mirror the attention-biasing effects of long-term priority cues like threat and self. Further work shows that goal-driven attention indeed reflects the motivational value of goal-relevant information. For instance, when the goal is to win as many tokens as possible, stimuli relevant to winning a high number of tokens attract attention over stimuli that are relevant to winning a low number of tokens (Vogt et al., 2011).

To examine the relative importance of temporary and long-term goals, Vogt et al. (2013) modified their spatial cueing task to include presentation of both temporary goalrelevant and threatening images. In the attention task, two images were presented in parallel 
as cues, with a dot probe appearing in one of the locations immediately after the image offset. Image pairs comprised combinations of four images: two threatening pictures (e.g., attacking snake, dog) and two neutral pictures (e.g., book, mushrooms). As in Vogt et al.'s (2010) study, the dot probe spatial cueing trials alternated with a goal detection task, in which each participant was assigned one of the four pictures as a goal image. Response time on the dot probe trials revealed an attentional bias toward goal images (whether these were threatening or neutral), but no congruence effect for threatening pictures when these were not participants' goal image (see also Brown et al., 2020; Vogt, et al., 2017). This pattern suggests that temporary goals override the effects of threatening stimuli; in other words, temporary goals sit atop this particular attentional hierarchy.

Given Vogt et al.'s (2013) findings, it is important to follow Experiment 1 with an examination of the relative importance of self cues in the context of temporary goals. Both self-relevant and goal-relevant cues over-ride the attentional bias that is usually associated with threatening cues (Exp. 1; Vogt et al., 2013). However, whether these same temporary goals also outweigh the attention-biasing effects of self-cues, or these are prioritized above short-term goals, is an empirical question. The purpose of Experiment 2 was to investigate this issue by modifying Vogt et al.'s (2013) design, to compare the effects of self goal images paired with angry and neutral images against angry and neutral goal images paired with selfimages. We examined whether associating images with temporary goals made self, angry, or neutral faces more attention-biasing, using a similar dot probe task to Experiment 1 . This allowed us to test whether temporary goals would override attention to the self-cues that were dominant on Exp 1, as well as exploring whether the effects of temporary goals are influenced by the type of stimulus to which they are assigned (e.g., i.e., self-cue goals vs. non-self cue goals). 


\section{Experiment 2: Self vs. Angry Goals}

The design of this experiment follows Vogt et al. (2013), in which a dot probe task was used in combination with a goal inducing task. Vogt et al. presented two threatening and two neutral images per participant, one of which was the goal image for each participant. In Experiment 2, we replicated this design but added a condition in which self-images replaced the threatening images from Vogt et al. (2013). To match Experiment 1, we used faces for the self, threatening and neutral images, with a front-facing and 3/4-facing image of each face included to allow a goal and non-goal version of the same identity to be presented.

Participants were assigned to one of four between-subjects conditions to examine the relative effects of self-cues and temporary goals on attention allocation: Self goal vs. angry, Self goal vs. neutral, Angry goal vs. self and Neutral goal vs. self. This design allowed us to test whether attention to self cues depends on their relevance to a temporary goal as well as the valence of the competing stimulus. (Note that the contrast of interest was between self and non-self (i.e., neutral/angry) goals, so we did not include an additional neutral goal vs. angry goal condition; see Vogt et al., 2013 for the results of this latter contrast.) In each condition, participants were presented with image pairs comprising the goal image, a nongoal image of the same identity, and two images of another identity. These different trial types allowed us to determine whether goal images bias attention independent of the comparison image (e.g., whether self-goal images override attention to non-goal self images, and to angry images of another person), or whether some non-goal images can override goal images (e.g., if self images are paired with angry goal images, which is most likely to attract attention?). Following the results of Vogt et al., (2013) it was expected that temporary goals would attract attention more effectively than either self or angry cues. However, following Experiment 1 it was expected that self-goal images would elicit the strongest attention allocation effects. 


\section{Method}

\section{Participants and Design.}

Sixty-nine female participants $\left(M_{\mathrm{age}}=20.5\right.$ years, $\left.S D_{\mathrm{age}}=1.7\right)$ at the University of Aberdeen participated in the experiment. A G*Power sample size calculation based on the effect size of $d=1.14$ (threatening vs. goal-relevant stimuli, Vogt et al., 2013), an alpha of 0.05 and power of 0.80 gave a required minimum sample size of 45 . Further, using the effect size provided by Liu et al. (2020) for trials comparing threatening to goal-relevant stimuli, $\eta 2 p=0.49$, the minimum sample size should be 44 , at an alpha of 0.05 and power of 0.80 . Using the effect size provided by Vogt et al. (2017; trials comparing threatening to goalrelevant (safety) stimuli, Experiment 1), $d=0.93$, the minimum sample size should be 64 at an alpha of 0.05 and power of 0.80 (required sample per group x 4). Stopping rule was completion of weekly participation sign ups once an appropriate sample size was reached. All participants had normal or corrected-to-normal vision and were naive as to the purpose of the experiment.

The experiment had four between-subjects conditions: Self goal vs. neutral, Self goal vs. angry, Neutral goal vs. self, and Angry goal vs. self. The repeated measures were Image Pairing (Goal image \& non-goal different identity image pairings vs. Goal image \& non-goal same identity image pairings) and Congruence (congruent vs. incongruent). As in Experiment 1, the dependent variable was response latency to the dot probe. Ethical approval was provided by the School of Psychology Ethics Committee at the University of Aberdeen.

\section{Apparatus and Materials.}

The experiment was programmed using the EPrime 2.0 software package that was implemented on an HP Elite Desk PC a 19-inch LCD monitor. All stimuli were presented 
against a black background.

\section{Face images}

Two self images were taken of each participant (a front and $3 / 4$ face view) with a light blank background. For the non-self images, we used the faces of two unfamiliar young, white, female models from the Radboud Face Database (database IDs: $01 \&$ 58; Langner, et al., 2010), which are presented on a light blank background. We used a front and $3 / 4$ face view of each model with a neutral and an angry expression. Each face image was $650 \mathrm{x} 482$ pixels in size and in full color. The designation of the face images as goal images was counterbalanced across participants, with each participant only having one goal face throughout (e.g., $3 / 4$ angry face, or front self face).

\section{Dot probe and goal tasks.}

Each trial in the dot probe task started with the presentation of a center-screen black fixation cross ( $5 \mathrm{~mm}$ high) on a white square, flanked above and below by a white box (4.6 $\mathrm{cm}$ high x $6.1 \mathrm{~cm}$ wide; see Figure 3). The middle of each of box was $4.6 \mathrm{~cm}$ from the fixation cross. Cues and probes were presented within the boxes. After $500 \mathrm{~ms}$, a picture was presented simultaneously in each box for $350 \mathrm{~ms}$. Immediately after picture offset, a dot

probe consisting of a small black square $(0.5 \mathrm{~cm} \times 0.5 \mathrm{~cm})$ appeared in the center of one of the boxes. Participants were required to indicate the probe location by pressing one of two keys ('4', '5' on the number pad) with index and middle finger of the right hand. Distribution of keys to probe locations was counterbalanced across participants. A trial ended after a response was registered or $1500 \mathrm{~ms}$ had elapsed since the onset of the probe.

A trial in the goal inducing task started with the appearance of one picture from the set of face images used in the dot probe task being presented in the middle of the screen for 
$250 \mathrm{~ms}$, after which it was replaced by a red question mark ( $8 \mathrm{~mm}$ high). A trial ended with a response (only required if the image matched the designated goal picture) or when $2000 \mathrm{~ms}$ had elapsed since the onset of the question mark. Responses required pressing the spacebar with the left hand. Correct reactions to the goal-relevant picture were followed by a feedback screen indicating that the reaction was correct. Incorrect reactions (i.e., no reaction) to the goal-relevant picture and incorrect reactions to the other pictures (i.e., pressing of the spacebar) were followed by error feedback that was presented for $200 \mathrm{~ms}$.

Figure 3: Experiment 2 trial schematic

\section{Dot probe task}
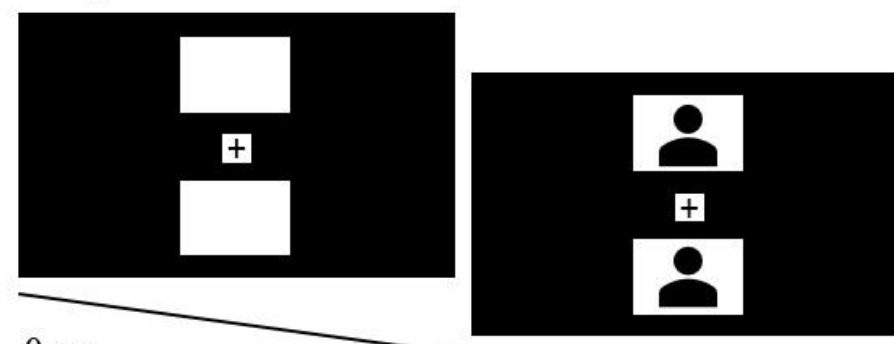

$0 \mathrm{~ms}$
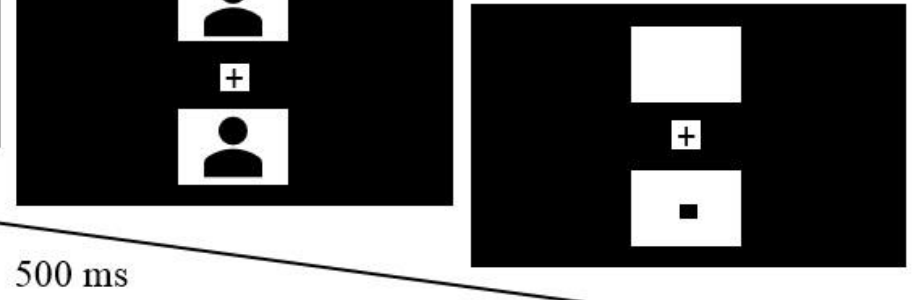

$850 \mathrm{~ms}$

Goal inducement task

$2350 \mathrm{~ms}$ (max)
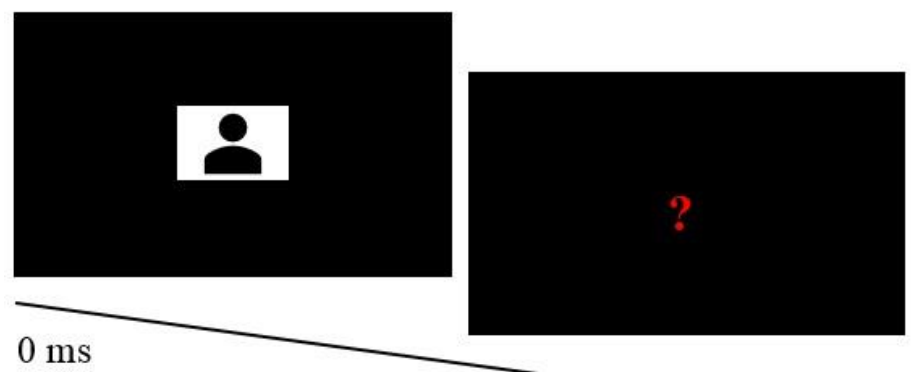

$250 \mathrm{~ms}$

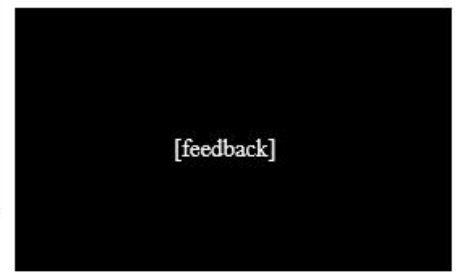

$2250 \mathrm{~ms}(\max )$

$2450 \mathrm{~ms}$ (max)

\section{Procedure.}

\section{Practice phase.}

As in Exp 1, participants were told they would be completing an image processing task and that one image would be their own face, requiring a photograph to be taken. After 
the participant's photograph was taken and uploaded to the programme, they were invited to sit at a table with the PC and keyboard, approximately 60 am away from the screen. All instructions were presented on the screen. For the dot probe task, participants were asked to maintain attention at the fixation cross and to respond as quickly and as accurately as possible to the probe location. For the goal task, instructions informed participants that after responding to the probe, a single picture would be presented in the middle of the screen. If this picture was the goal-relevant picture, they should press the spacebar with the left hand when the question mark appeared. Instructions for the goal task emphasized that speed was not important in this task. The task began with a practice phase of 15 trials with different pictures to the test phase to prevent habituation.

\section{Test phase.}

Before the test phase, participants were shown their designated goal picture (e.g., $3 / 4$ angry face), and were told that this picture would be used throughout the test phase. Instructions informed participants that they would win ten points for correctly indicating the presence of the goal-relevant picture during the goal trials. In line with previous research, we did not give information on points won in total or similar as winning points itself seems to be sufficiently motivating (cf. Vogt et al., 2010, 2013). Trials alternated between goal and dot probe task, separated by an inter-trial interval of $600 \mathrm{~ms}$.

In the dot probe task, participants in each Goal Condition were presented with paired combinations of four images (see Table 1). For example, if a participant was in the Self-goal vs. neutral condition, they were presented with pairings comprising a self (front), self ( $3 / 4)$, neutral (front), and neutral (3/4) image. If the participants' goal image was the self-front image, there would be three non-goal images - one of the same person but a different view (self $3 / 4$ image), and two neutral images of a different person (front and $3 / 4$ views). Each of the 
six possible pairing combinations of these images was presented 32 times, resulting in 192 trials in the dot probe task. Each of the four images in the condition was presented on 48 trials, so goal images appeared on 48 of the trials and 144 trials contained no goal images. All images appeared equally often in the goal and dot probe task.

Trials of goal and dot probe task alternated, with an inter-trial interval of $600 \mathrm{~ms}$. Each picture category was presented on half of the trials in the top location and on the other half in the bottom location and the probe was presented on the location of pictures of that category on half of the trials. The order of the different trials of both tasks was determined randomly for each participant. The order of the dot probe task trials and the goal task trials was determined independently. Subsequently, the cue pictures that were presented in a dot probe trial were not predictive of the picture that would appear in the consecutive trial of the goal task.

This study was not pre-registered but the full dataset can be accessed at https://osf.io/4re5b/.

\section{Results}

Performance in the goal task was high (94.9\% accuracy), suggesting participants successfully maintaining an active goal of monitoring for the relevant image. Following Vogt et al. (2013), trials with errors in the dot probe task were removed from the data (6.9\%). The data of three participants were excluded because their error rates were higher than the sample's mean error \% plus three times the standard deviation of the sample's error \%. RTs faster than $150 \mathrm{~ms}$ and slower than $1000 \mathrm{~ms}$ were considered as outliers and discarded from the analyses (1.72\%; cf. Vogt et al., 2013), then mean RT per condition was calculated as the dependent variable (see Table 2).

The Goal condition data were collapsed across participants whose goal images were 
front and $3 / 4$ images of the same face (e.g., in the 'Self-goal vs. neutral' condition, data were collapsed across participants whose goal image was a front self image and those whose goal image was a 3/4 self image). Further, because both images (front and 3/4) of the person who was not featured in the goal image were identical in terms of experimental condition (i.e., 'Non-goal (different identity)'), their mean response times were also collapsed. However, the means of the two images (front and $3 / 4$ ) of the person who did feature in the goal image were not collapsed because they were in different experimental conditions, one being the goal image and the other being a non-goal image of the same identity.

We have restricted our primary reporting of results to the analyses that address the effects of theoretical interest; that is, comparing responses to targets on trials where goal images were paired with different non-goal images, when the goal image was either congruent or incongruent with the dot probe.

To establish whether images associated with temporary goals capture attention more than self face images, we analysed the mean reaction times using a 4 (Goal Condition: self goal \& neutral vs. self goal \& angry vs. neutral goal \& self vs. angry goal \& self) x 2 (Image Pairing: goal image \& non-goal image of different identity vs. goal image \& non-goal image of goal identity) X 2 (Congruence: congruent vs. incongruent) mixed factorial ANOVA with Goal Condition as the only between-subjects factor. For these analyses, congruent trials are trials in which the probe replaced the goal image, whereas incongruent trials are trials in which the probe replaced the paired non-goal image (Vogt et al., 2013).

The analysis revealed significant main effects of Goal Image Pairing $[F(1,62)=$ $11.23, p=.001, \eta^{2} \mathrm{p}=.153$ ], with faster RTs when the goal image was paired with a non-goal image of a different identity $(M=434.2 \mathrm{~ms})$ vs. the same identity $(M=445.1 \mathrm{~ms})$. There was also a main effect of Congruence $\left[F(1,62)=80.24, p<.001, \eta^{2} \mathrm{p}=.564\right]$, with faster 
responses when the probe was congruent $(M=434.3 \mathrm{~ms})$ than incongruent $(M=460.0 \mathrm{~ms})$ with the goal image. There was no evidence of a main effect of Goal Condition $[F(3,62)=$ $\left.1.39, p=.256, \eta^{2} \mathrm{p}=.063\right]$ or a Goal Condition X Congruence interaction $[F(3,62)=2.04, p$ $\left.=.117, \eta^{2} \mathrm{p}=.090\right]$, but there were significant two-way interactions of Goal Condition $\mathrm{X}$ Image Pairing $\left[F(3,62)=3.33, p=.025, \eta^{2} \mathrm{p}=.139\right]$ and Image Pairing X Congruence $[F(1$, 62) $\left.=13.79, p<.001, \eta^{2} \mathrm{p}=.182\right]$, and a marginal three-way interaction of Goal Condition $\mathrm{X}$ Image Pairing $X$ Congruence $\left[F(3,62)=2.62, p=.059, \eta^{2} p=.113\right]$. Given their central theoretical importance, we further examined the interaction patterns by analysing the data from each Goal Condition using four separate 2 (Image Pairing: goal image \& non-goal image of different identity vs. goal image \& non-goal image of goal identity) X 2 (Congruence: congruent vs. incongruent) repeated measures ANOVAs (see below). 


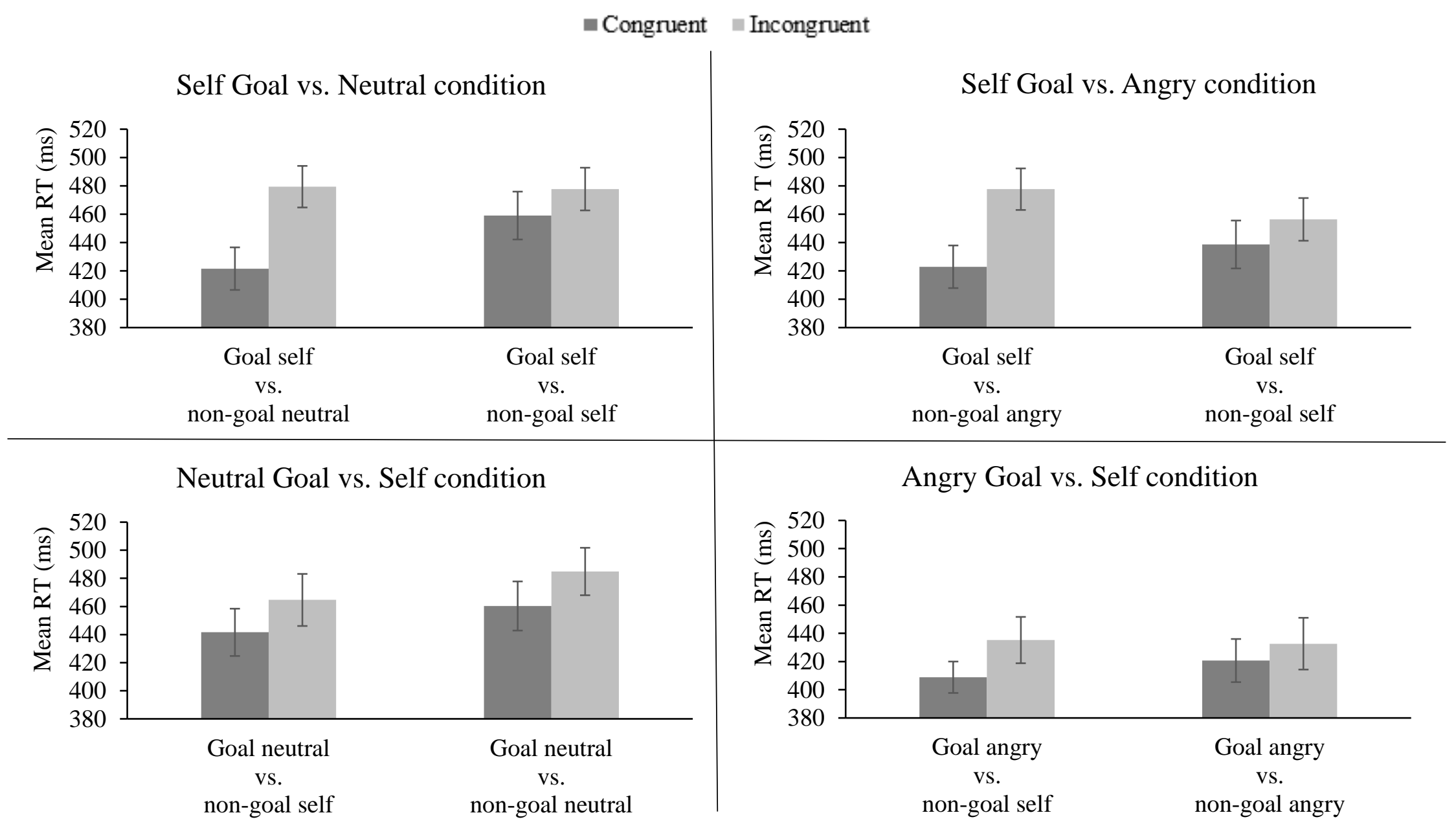

Figure 4: Mean dot probe reaction time in each goal condition. Congruent trials are those when the target appears at the same location as the goal image; incongruent trials are those when the target appears at the same location as the other image.

Error bars represent one standard error of the mean. 
Self Goal vs. Neutral: Data for congruent and incongruent trials for both goal image pairings in the Self Goal vs. Neutral condition can be seen in Figure 4 (top-left panel). These data were analysed with a 2 (Image Pairing: goal-self vs. non-goal neutral pairing, goal-self vs. non-goal self pairing) X 2 (Congruence: goal congruent vs. goal incongruent) repeated measures ANOVA. This revealed a main effect of Congruence $\left[F(1,17)=37.68, p<.001, \eta^{2} \mathrm{p}=.689, d_{\mathrm{z}}=1.41\right]$, such that participants were faster to respond on goal congruent trials $(M=440 \mathrm{~ms})$ relative to goal incongruent trials $(M=479 \mathrm{~ms})$. There was also a main effect of Image Pairing $[F(1,17)=$ $\left.14.87, p=.001, \eta^{2} \mathrm{p}=.467\right]$, such that participants were faster to respond when goal self images were paired with non-goal neutral images $(M=451 \mathrm{~ms})$, relative to when they were paired with non-goal self images $(M=468 \mathrm{~ms})$.

These main effects were subsumed by a significant Image Pairing X Congruence interaction $\left[F(1,17)=12.64, p=.002, \eta^{2} \mathrm{p}=.426\right]$. To determine whether there was evidence of attentional bias towards goal images, we began by running paired samples t-tests comparing trials when the goal image was congruent relative to trials when it was incongruent for each Image Pairing level. There was a significant goal image bias in the Goal-self vs. neutral pairing condition; On trials when a goal self image appeared with a non-goal neutral image, people were significantly faster when the goal-self image was congruent $(M=422 \mathrm{~ms})$ than incongruent $(M=$ $\left.479 \mathrm{~ms} ; t(17)=9.29, p<.001, \eta^{2} \mathrm{p}=.836, d_{\mathrm{z}}=2.13\right)$. On trials when a goal self image appeared with a non-goal self image, there was a similar but non-significant trend for people to respond faster when the goal-self image was congruent $(M=459 \mathrm{~ms})$ than incongruent $(M=478 \mathrm{~ms} ; t(17)$ $\left.=1.87, p=.078, \eta^{2} \mathrm{p}=.171, d_{\mathrm{z}}=0.44\right)$.

We further explored this interaction to examine whether the size of the attentional bias towards goal self faces was dependent on the face image with which it was paired.. We did this by calculating congruency difference scores (i.e., incongruent trials minus congruent trials) for 
each Image Pairing and comparing these with a paired samples t-test. This revealed there was significantly stronger bias towards goal self images when they were paired with non-goal neutral images $(M \operatorname{diff}=58 \mathrm{~ms})$ relative to when they were paired with non-goal self images $(M \operatorname{diff}=$ $\left.19 \mathrm{~ms} ; t(17)=3.56, p=.002, \eta^{2} \mathrm{p}=.426\right)$.

Self Goal vs. Angry: Data for congruent and incongruent trials for both goal image pairings in the Self Goal vs. Angry condition can be seen in Figure 4 (top-right panel). These data were analysed with a 2 (Image Pairing: goal-self vs. non-goal angry pairing, goal-self vs. non-goal self pairing) X 2 (Congruence: goal congruent vs. goal incongruent) repeated measures ANOVA. The analysis revealed a main effect of Congruence $\left[F(1,15)=33.15, p<.001, \eta^{2} \mathrm{p}=.688, d_{\mathrm{z}}=\right.$ 1.42], such that participants were faster to respond on goal congruent trials $(M=431 \mathrm{~ms})$, relative to goal incongruent trials $(M=467 \mathrm{~ms})$. There was no main effect of Image Pairing $[F(1,15)=$ $\left..563, p=.465, \eta^{2} \mathrm{p}=.036\right]$.

The main effect of Congruence was subsumed by a significant Image Pairing X Congruence interaction $\left[F(1,15)=11.09, p=.005, \eta^{2} \mathrm{p}=.425\right]$. To determine whether there was evidence of attentional bias towards goal images, we first ran paired samples t-tests comparing trials when the goal image was congruent relative to trials when it was incongruent for each Image Pairing level. There was a significant goal image bias in the Self-goal vs. Angry pairing condition; on trials when a goal self image appeared with a non-goal angry image, people were significantly faster when the goal-self image was congruent $(M=423 \mathrm{~ms})$ than incongruent $(M=$ $\left.478 \mathrm{~ms} ; t(15)=5.71, p<.001, \eta^{2} \mathrm{p}=.685, d_{\mathrm{z}}=1.43\right)$. Similarly, on trials when a goal self-image appeared with a non-goal self image, people were significantly faster when the goal self image was congruent $(M=439 \mathrm{~ms})$ than incongruent $\left(M=456 \mathrm{~ms} ; t(15)=2.53, p=.023, \eta^{2} \mathrm{p}=.298, d_{\mathrm{z}}\right.$ $=0.61)$. 
As was the case in the Goal self vs. neutral analysis, we further explored this interaction using a paired samples t-test of congruency difference scores. This revealed there was a significantly stronger bias towards goal self images when they were paired with non-goal angry images $(M \operatorname{diff}=55 \mathrm{~ms})$ relative to when they were paired with non-goal self images $(M \operatorname{diff}=$ $\left.18 \mathrm{~ms} ; t(17)=3.56, p=.002, \eta^{2} \mathrm{p}=.426\right)$.

Neutral Goal vs. Self: Data for congruent and incongruent trials for both goal image pairings in the Neutral goal vs. Self condition can be seen in Figure 4 (bottom-left panel). These data were analysed with a 2 (Image Pairing: Goal-neutral vs. self pairing, Goal-neutral vs. non-goal neutral pairing) X 2 (Congruence: goal congruent vs. goal incongruent) repeated measures ANOVA. The analysis revealed a main effect of Congruence $\left[F(1,15)=24.43, p<.001, \eta^{2} \mathrm{p}=.620, d_{\mathrm{z}}=\right.$ 1.26], such that participants were faster to respond on goal congruent trials $(M=451 \mathrm{~ms})$, relative to goal incongruent trials $(M=475 \mathrm{~ms})$. There was also a main effect of Image Pairing $[F(1,15)$ $\left.=13.98, p=.002, \eta^{2} \mathrm{p}=.482\right]$, such that participants were faster to respond when goal-neutral images were paired with non-goal self images $(M=451 \mathrm{~ms})$, relative to when goal-neutral images were paired with non-goal neutral images $(M=473 \mathrm{~ms})$. However, there was no evidence of a significant Image Pairing X Congruence interaction $\left[F(1,15)=0.21, p=.886, \eta^{2} p=.001\right]$.

Angry Goal vs. Self: Data for congruent and incongruent trials for both goal image pairings in the Angry Goal vs. Self condition can be seen in Figure 4 (bottom-right panel). These data were analysed with a 2 (Image Pairing: Goal-angry vs. self pairing, Goal-angry vs. non-goal angry pairing) X 2 (Congruence: goal congruent vs. goal incongruent) repeated measures ANOVA. The analysis revealed a significant main effect of Congruence $\left[F(1,15)=5.22, p=.037, \eta^{2} \mathrm{p}=\right.$ $\left..258, d_{\mathrm{z}}=0.57\right]$, such that participants were faster to respond on goal congruent trials $(M=$ 
$415 \mathrm{~ms})$, relative to goal incongruent trials $(M=434 \mathrm{~ms})$. There was no main effect of Image Pairing $\left[F(1,15)=.280, p=.605, \eta^{2} p=.018\right]$ and no Image Pairing X Congruence interaction $\left[F(1,15)=.902, p=.357, \eta^{2} \mathrm{p}=.057\right]$

Further analysis of the effects of comparison image: The main effects of image pairing reported above suggest that the identity of comparison image (i.e., same identity as the goal image vs. different identity to the goal image) influences the impact of the goal image on attention. To examine the relative effects of self, angry and neutral goals in the absence of same identity faces, we assessed attentional bias when goal images were paired with the other identity faces only (see Figure 5). We analysed the mean reaction time difference scores (i.e., goal incongruent trials minus goal congruent trials) using a univariate ANOVA with a single between-subjects factor (Goal Condition: Self goal vs neutral, Self goal vs. angry, Neutral goal vs. self, Angry goal vs. self). This analysis revealed a significant main effect $[F(3,62)=4.54, p$ $\left.=.007, \eta^{2} \mathrm{p}=.177\right]$

Pairwise comparisons indicated that the size of the attentional bias was significantly larger when the goal was a self image: the Self Goal vs. Neutral condition $\left(M_{\text {diff }}=58 \mathrm{~ms}\right)$ exceeded both the Neutral Goal vs. Self $\left(M_{\text {diff }}=23 \mathrm{~ms} ; p=.006\right)$ and Angry Goal vs Self conditions $\left(M_{\text {diff }}=26 \mathrm{~ms} ; p=.012\right)$, and similarly the Self Goal vs. Angry condition $\left(M_{\text {diff }}=\right.$ $55 \mathrm{~ms})$ difference significantly exceeded the Neutral Goal vs. Self $(p=.014)$ and Angry Goal vs. Self conditions $(p=.027)$. No other comparisons approached significance. This pattern suggests that self-goal cues were more effective at capturing attention away from angry and neutral images, than angry-goal or neutral-goal cues were at capturing attention away from self-images. 


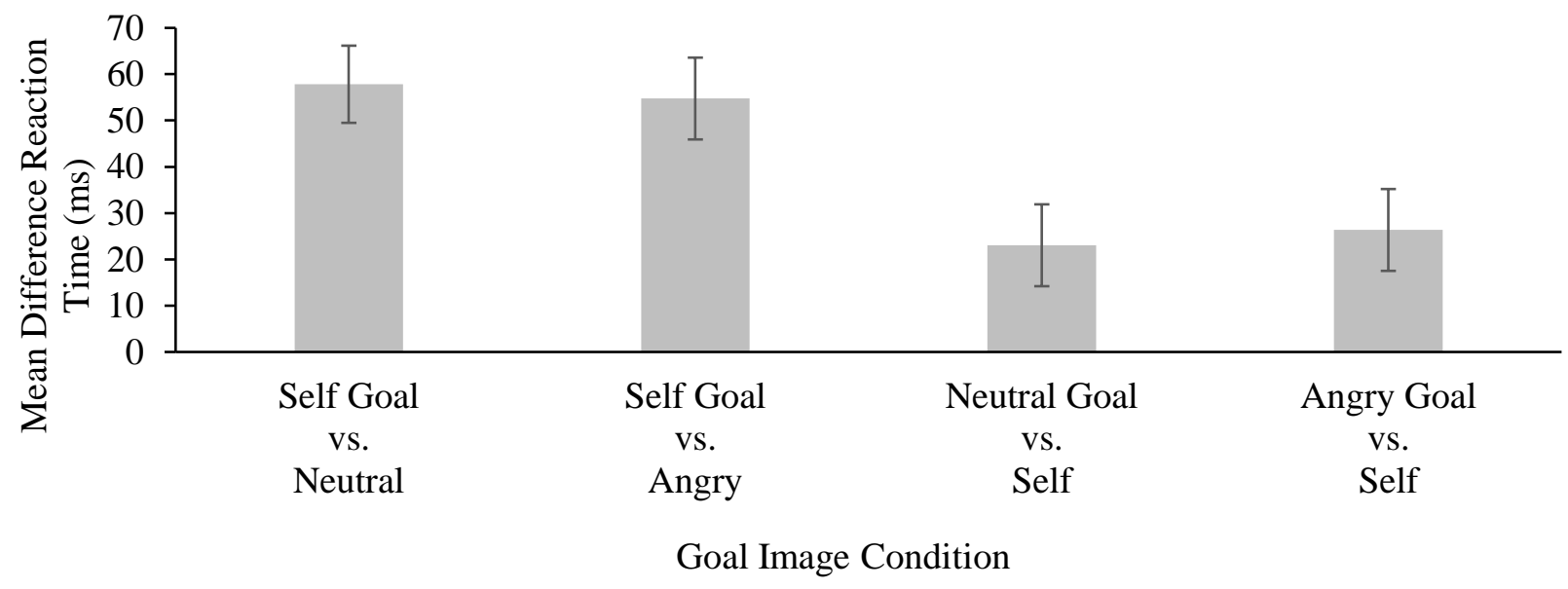

Figure 5: Mean dot probe reaction time difference scores (i.e., goal incongruent trials minus goal congruent trials) by condition. Error bars represent one standard error of the mean.

Non-goal trials: Finally, trials with no goal image present were examined separately. The results of the analysis of the non-goal vs. non-goal data complement and do not contradict the pattern of effects for the goal vs. non-goal data. Specifically, participants showed significantly greater bias towards non-goal self images in the Self goal vs neutral condition ( $M$ diff $=28 \mathrm{~ms})$ than towards non-goal neutral images in the Neutral goal vs. self condition ( $M$ diff $=6 \mathrm{~ms} ; p=.016)$, or nongoal angry images in the Angry goal vs. self condition ( $M$ diff $=9 \mathrm{~ms} ; p=.042)$. Similarly, participants showed significantly greater bias towards non-goal self images in the Self goal vs. angry condition $(M \operatorname{diff}=28 \mathrm{~ms})$ than towards non-goal neutral images in the Neutral goal vs. self condition ( $M$ diff $=6 \mathrm{~ms} ; p=.028)$, and showed a non-significant trend in the same direction relative to non-goal angry images in the Angry goal vs. self condition ( $M$ diff $=9 \mathrm{~ms} ; p=.067)$. Together, these patterns suggest that when participants' goal image was of their own face, this created a particularly strong attention bias for other (non-goal) images of themselves, relative to the attention bias associated with having a goal image of an unfamiliar person. 


\section{Discussion}

Experiment 2 sought to establish whether images associated with temporary goals capture attention more than self face images. In addition, it aimed to examine whether goal images of self faces result in greater attentional bias than do goal images of angry or neutral faces. There was evidence for both of these proposals. People showed attentional bias towards goal images, relative to non-goal images, even when the non-goal images were self faces. In other words, such was the influence of temporary goals that when angry and neutral face images were made goalrelevant, they were able to bias attention away from self face images in a way that the same images were unable to do in Experiment 1. However, when self face images were the focus of temporary goals, this resulted in significantly greater attentional bias than did either neutral or angry goal images of another person.

The finding that temporary goals influence attention allocation replicates previous results obtained using for neutral and threatening non-face cues (e.g., attacking snake, mushrooms; Vogt et al., 2013). In Vogt et al.'s study, it was found that there was an attentional bias to temporary neutral or threatening goal images when these were paired with neutral or threatening non-goal images. This pattern was exactly replicated by the dominance of goals in the current findings, which also provide an extension to Vogt et al. by demonstrating a temporary goals effect for a positive stimulus type.

An additional pattern that emerged was that for two of the four conditions there was a slower response time overall when the goal image was paired with the non-goal image of the same identity. This fits the intuitive reasoning that it is more difficult to differentiate between two images of the same identity, than between two different identities. Of greater theoretical interest, in the two self-goal conditions (i.e., Self-goal vs. Angry, Self-goal vs. Neutral), this effect was qualified by an interaction with congruency; there was a greater attention bias to the 
goal image when it was paired with an image of a different identity (i.e., an angry or neutral image) than when it was paired with another, non-goal self image. The equivalent interaction did not emerge when the goal was neutral or angry, so whether the other image was the same or a different (unfamiliar) identity as the goal image did not impact on the attention bias to the goal image. Corresponding with this pattern, within trials in which the non-goal image was a different identity to the goal image, attentional bias to the goal image was greatest when that image was of self, relative to angry or neutral. . These patterns suggest that while the attentiongrabbing effects of self images sit below temporary goals in the attentional hierarchy, they provide stronger competition than angry or neutral images of other people.

\section{General Discussion}

In the real world, the attention system is required to navigate multiple cues in the environment that may be competing for attention. The current study was designed to assess the results of this competition, between cues already known to attract attention: threatening images, self-images, and those relevant to current goals. Across two experiments, self-images were found to bias attention more than angry or neutral images (Exp. 1, Exp. 2), and temporary goals were found to outweigh the attentional bias toward self-images (Exp 2). However, the biasing effects of goal images were heightened when the goal was a self-image (Exp. 2). On the basis of the cues included in the current study, temporary goals would appear to be prioritized within the attentional hierarchy, with self-cues exerting a secondary influence that is stronger than angry and neutral cues.

The prominence of temporary goals replicates Vogt et al. (2013)'s study using different angry and neutral goal cues, and speaks to the importance of the dynamic qualities of the attention system. Current goals are prioritized, even when they comprise short-term goals on a 
non-critical laboratory task. This is an interesting finding because it is somewhat counterintuitive; as Vogt et al point out, it might be predicted that 'hard-wired' biases toward specific stimulus types with a survival advantage would have priority. Both responses to threat cues and self-processing biases are argued to meet this adaptive criterion (see Cunningham, 2016; Cunningham et al., 2013; Öhman \& Mineka, 2001), so these stimuli should be perpetually capturing attention. However, the prioritisation of temporary goals shows the adaptability of the attention system: the most important cue is that relating to the current task, and when there is competition for attention, the current goal is prioritized.

Next on the attentional hierarchy behind temporary goals were self cues. In both experiments (all Exp 1 data, non-goal trials data in Exp 2), self images were associated with higher attentional bias than equivalent angry images. Further, in Exp 2 self-goal cues were more effective at capturing attention away from angry and neutral non-goal images, than angry-goal or neutral-goal cues were at capturing attention away from non-goal self-images.. This may reflect the stability of self as a perpetual goal. An image on one's own face is self-relevant in any context, whether processing cues in an attention task or catching sight of oneself in a mirror. Self is perpetually activated because every aspect of the internal and external environment processed is done so from the same self-perspective, regardless of context (Conway, 2005). In contrast, threatening images can be rendered more or less threatening by the processing context. For example, an angry face in an experimental task may evoke a weaker response than the same face being encountered on the street. Accordingly, while some studies have reported attentional biases to threatening images (Vuilleumier \& Huang, 2009; Yiend, 2010), this effect does not always emerge, especially when attentional resources are being divided between tasks (e.g., Van Dillen $\&$ Koole, 2009) or between different attention-capturing cues (e.g., Vogt et al., 2013). Consistent with this reasoning, the current findings suggest that when threatening cues are in competition 
with other biasing stimuli, they are less effective at capturing attention.

It is important to consider whether the lack of an attentional bias toward angry cues reported here is due to angry faces being insufficiently threatening as a stimulus type (e.g., relative to an image of an attacking predator). Importantly, an examination of attentional biases in the sub-group of Exp. 1 participants who did show an attentional bias toward angry images revealed that even in those participants, there was a stronger bias to self cues than angry cues. This suggests that even when angry stimuli evoked a measurable threat response in attention, this was secondary to the attentional response to self-cues in the same participants. An identical pattern was found by Vogt et al. (2013), whereby attention was oriented to neutral goals rather than towards a variety of threatening cues (e.g., attacking predator image, aversive noise). This converging evidence suggests that when there is competition for attentional resources threatening cues are less effective at influencing attention allocation than temporary goals or self-relevant cues.

An interesting product of the attentional-capturing potential of goals and self-cues is the pattern that arises when current goals are self-relevant. In the current study there was a significantly greater attentional bias when the goal was a self cue (paired with angry and neutral non-goal cues) than when the goal was a neutral or angry cue (paired with self non-goal cues). There was no difference between the relative attentional pull of angry and neutral cues (replicating Vogt et al., 2013). It is possible that this pattern emerged because attention-capturing biases are additive - i.e., if temporary goal and self-cues are both effective at attracting attention, then self-goals are an even stronger type of goal cue. Further, it is possible that because selfimages are highly familiar relative to the images of the strangers used in the current study, participants found it easier to identify the goal-image when it was self (i.e., to distinguish between the two views of the self image than an image of a stranger). However, a more 
parsimonious explanation is that the pattern is driven by competition with the non-goal cues. Both angry and neutral cues were paired with self-cues, which are highly effective at capturing attention. In contrast, self-goals were paired with angry and neutral cues, neither of which offered effective competition for attentional resources. This again is consistent with the purported relative dominance of self-cues in the attentional hierarchy.

An important caveat to the current findings is that while we have examined the relative positions of goal, self and threat cues in the attentional hierarchy, this is by no means an exhaustive list of attention-capturing stimuli. The relative biasing effects of other cue types such as sudden-onset exogenous cues and those with particular perceptual qualities, emotional arousal or attractiveness should all be examined in future research. A fuller examination of how these stimulus types operate when in competition within the visual environment will provide a clearer picture of adaptive processing in the attention system. Future research should also compare goal stimuli to comparison stimuli that have to be searched or require a response (cf. Sha \& Liang, 2016; Qu et al., 2017) though previous studies have excluded action relevance as alternative explanation (Godara et al., 2020; Vogt et al., 2017).

Further, the influence of demographic variables was not explored in the current studies. Indeed, in Exp. 2 only female participants were included for practical reasons, a potential limitation of this dataset. However, it is unlikely that gender would impact on the attention biases explored here, with a recent meta-analytic review concluding that there is no evidence for gender differences in responses to dot probe tasks that include threat cues (Campbell et al., 2017), and gender not being posited as a relevant theoretical factor in self-processing biases (e.g., see reviews by Humphreys \& Sui, 2016; Symons \& Johnson, 1997). As well as being majority female, the samples tested in the current study were also majority Western and Caucasian, reflecting the local student population. While race effects do not appear to be dominant in face- 
based dot probe tasks (see Correll et al., 2014), cultural differences may be influential in shaping the attentional hierarchy. In particular, some East Asian, collectivist cultures report lower selfbias than those shown in Western, individualistic cultures (e.g., Sparks et al., 2016; Zhang et al., 2020; Zhu et al., 2007). It would therefore be of interest to explore the attentional hierarchy in non-Western populations.

In conclusion, the findings of the current study suggest that the self does come first in the attentional hierarchy when competing with angry and neutral stimuli. However, if temporary goals become active these assume priority status; a pattern that is particularly strong if the temporary goal is self-relevant. These findings show that while cues associated with the self are dominant in determining attention allocation across aspects of the environment, the system is ultimately shaped by the perceiver's goal while negotiating that environment.

\section{References}

Bargh, J. A. (1982). Attention and automaticity in the processing of self-relevant information. Journal of Personality and Social Psychology, 43(3), 425-436. doi:10.1037/00223514.43.3.425. https://doi.org/10.1037/0022-3514.43.3.425

Bargh, J. A. \& Pratto, F. (1986). Individual construct accessibility and perceptual selection. Journal of Experimental Social Psychology, 22, 293-311. https://doi.org/10.1016/00221031(86)90016-8

Bar-Haim, Y., Lamy, D., Pergamin, L., Bakermans-Kranenburg, M. J., \& van IJzendoorn, M. H. (2007). Threat-related attentional bias in anxious and nonanxious individuals: A metaanalytic study. Psychological Bulletin, 133, 1-24. http://dx.doi.org/10.1037/00332909.133.1.1 
Becker, D. V., Anderson, U. S., Mortensen, C. R., Neufeld, S. L., \& Neel, R. (2011). The face in the crowd effect unconfounded: Happy faces, not angry faces, are more efficiently detected in single- and multiple-target visual search tasks. Journal of Experimental Psychology:

General, 140(4), 637-659. https://doi.org/10.1037/a0024060

Beggan, J. K. (1992). On the social nature of nonsocial perception: The mere ownership effect. Journal of Personality and Social Psychology, 62, 229-237. https://doi.org/10.1037/00223514.62.2.229

Berger, A., Henik, A., \& Rafal, R. (2005). Competition between endogenous and exogenous orienting of visual attention. Journal of Experimental Psychology: General, 134(2), 207221. https://doi.org/10.1037/0096-3445.134.2.207

Brédart, S., Delchambre, M., \& Laureys, S. (2006). One's own face is hard to ignore. Quarterly Journal of Experimental Psychology, 59, 46-52. https://doi.org/10.1080/17470210500343678

Brown, C. R., Berggren, N., \& Forster, S. (in press). Testing a goal-driven account of involuntary attentional capture by threat. Emotion. https://doi.org/10.1037/emo0000565

Campbell, A. \& Muncer, S. (2017). Sex differences in awareness of threat: A meta-analysis of sex differences in attentional orienting in the dot probe task. Personality and Individual Differences, 119, 181-184. https://doi.org/10.1016/j.paid/2017.07.04

Conway, M. A. (2005). Memory and the self. Journal of Memory and Language, 53, 594-628. https://doi.org/10.1016/j.jml.2005.08.005

Conway, M. A., \& Pleydell-Pearce, C. W. (2000). The construction of autobiographical memories in the self-memory system. Psychological Review, 107(2), 261288. https://doi.org/10.1037/0033-295X.107.2.261

Correll, J., Guillermo, S. \& Vogt, J. (2014). On the flexibility of attention to race. Journal of 
Experimental Social Psychology, 55, 74-79. https://doi.org/10.1016/j.jesp.2014205.13

Cunningham, S. J. (2016). The function of the self-attention network. Cognitive Neuroscience, 7, 21-22. https://doi.org/10.1080/17588928.2015.1075485

Cunningham, S. J., Brady-Van den Bos, M., Gill, L., \& Turk, D. J. (2013). Survival of the selfish: Contrasting self-referential encoding and survival processing. Consciousness and Cognition, 22, 237-244. https://doi.org/10.1016/j.concog.2012.12.005

Dannemiller, J. L. (2000). Competition in early exogenous orienting between 7 and 21 weeks. Journal of Experimental Child Psychology, 76(4), 253-274. https://doi.org/10.1006/jecp.1999.2551

Desimone, R., \& Duncan, J. (1995). Neural mechanisms of selective visual attention. Annual Review of Neuroscience, 18, 193-222. https://doi.org/10.1146/annurev.ne.18.030195.001205

E-Prime (Version 2.0) [Computer software]. Pittsburgh, PA: Psychology Software Tools. Everaert, J., Koster, E.H.W., Derakhshan, Nazanin (2012). The combined cognitive bias hypothesis in depression: a state-of-the-art. Clinical Psychology Review, 32(5), 413-424.

Faul, F., Erdfelder, E., Lang, A.-G., \& Buchner, A. (2007). G*Power 3: A flexible statistical power analysis program for the social, behavioral, and biomedical sciences. Behavior Research Methods, 39, 175-191.doi: 10.3758/bf03193146.

Folk, C. L., Remington, R. W., \& Johnston, J. C. (1992). Involuntary covert orienting is contingent on attentional control settings. Journal of Experimental Psychology: Human Perception and Performance, 18(4), 1030-1044. https://doi.org/10.1037/0096$\underline{1523.18 .4 .1030}$

Godara, M., Van Bockstaele, B., \& Wiers, R. W. (2020). Conflicting rewards: Effects of task goals on attention for alcohol cues. Cognition and Emotion, 34(4), 643-655. 
https://doi.org/10.1080/02699931.2019.1664996

Grubb, M.A., White, A.L., Heeger, D.J. \& Carrasco, M. (2015). Interactions between voluntary and involuntary attention modulate the quality and temporal dynamics of visual processing. Psychonomic Bulletin and Review, 22, 437-444. https://doi.org/10.3758/s13423-014-0698-y

Hahn, S., \& Gronlund, S. D. (2007). Top-down guidance in visual search for facial expressions. Psychonomic Bulletin \& Review, 14, 159-165. https://doi.org/10.3758/BF03194044

Harris, C. R. \& Pashler, H. (2004). Attention and the processing of emotional words and names: Not so special after all. Psychological Science, 15, 171-178. https://doi.org/10.1111/j.0956-7976.2004.01503005.x

Hodsoll, S., Viding, E., \& Lavie, N. (2011). Attentional capture by irrelevant emotional distractor faces. Emotion, 11(2), 346-353. https://doi.org/10.1037/a0022771

Humphreys, G. W. \& Siu, J. (2016). Attentional control and the self: The self-attention network (SAN). Cognitive Neuroscience, 7(1-4), 5-17. https://doi.org/10.1080/17588928.2015.1044427

Ito, T. A., Larsen, J. T., Smith, N. K., \& Cacioppo, J. T. (1998). Negative information weighs more heavily on the brain: The negativity bias in evaluative categorizations. Journal of Personality and Social Psychology, 75(4), 887-900. https://doi.org/10.1037/00223514.75.4.887

Kuhn, G., Pickering, A., \& Cole, G. G. (2016). "Rare” emotive faces and attentional orienting. Emotion, 16, 1-5. https://doi.org/10.1037/emo0000050

Lakens, D. (2013). Calculating and reporting effect sizes to facilitate cumulative science: a practical primer for t-tests and ANOVAs. Frontiers in Psychology, 4, 863. doi: 10.3389/fpsyg.2013.00863 
Langner, O., Dotsch, R., Bijlstra, G., Wigboldus, D.H.J., Hawk, S.T., \& van Knippenberg, A. (2010). Presentation and validation of the Radboud Faces Database. Cognition \& Emotion, 24(8), 1377-1388. https://10.1080/02699930903485076

Liu, Y., Wang, P. \& Wang, G. (2020). The priority of goal-relevant information and evolutionarily threatening information in early attention processing: Evidence from behavioral and ERP study. Scientific Reports 10, 8008. https://doi.org/10.1038/s41598$020-65062-5$

Luck, S. J., \& Vecera, S. P. (2002). Attention. In H. Pashler (Series Ed.) \& S. Yantis (Volume Ed.), Stevens' Handbook of Experimental Psychology: Vol. 1. Sensation and Perception (3rd ed., pp. 235-286). New York: Wiley.

MacLeod, C., Mathews, A., \& Tata, P. (1986). Attentional bias in emotional disorders. Journal of Abnormal Psychology, 95, 15-20. https://doi.org/10.1037/0021-843X.95.1.15

Macrae, C. N., Visokomogilski, A., Golubickis, M., Cunningham, W. A., \& Sahraie, A. (2017). Self-relevance prioritizes access to visual awareness. Journal of Experimental Psychology: Human Perception and Performance, 43(3), 438-443. https://doi.org/10.1037/xhp0000361

Mogg, K., Bradley, B. P., Hyare, H., \& Lee, S. (1998). Selective attention to food-related stimuli in hunger: are attentional biases specific to emotional and psychopathological states, or are they also found in normal drive states? Behaviour Research and Therapy, 36(2), 227237. https://doi.org/10.1016/S0005-7967(97)00062-4

Mogg, K. \& Bradley, B. P. (1999). Some methodological issues in assessing attentional biases for threatening faces in anxiety: a replication study using a modified version of the probe detection task. Behaviour Research and Therapy, 37, 595-604. https://doi.org/10.1016/S0005-7967(98)00158-2 
Moray, N. (1959). Attention in dichotic listening: Affective cues and the influence of instructions. Quarterly Journal of Experimental Psychology, 11, 56-60. https://doi.org/10.1080/17470215908416289

Notebaert, L., Crombez, G., Van Damme, S., De Houwer, J., \& Theeuwes, J. (2011). Signals of threat do not capture, but prioritize, attention: A conditioning approach. Emotion, 11, 8189. https://doi.org/10.1037/a0021286

Oberauer, K. 2019. Working memory and attention - a conceptual analysis and review. Journal of Cognition, 2(1):36, 1-23. https://doi.org/10.5334/joc.58

Öhman, A. (2007). Has evolution primed humans to "beware the beast"? Proceedings of the National Academy of Sciences, 104(42), 16396-16397. https://doi.org/10.1073/pnas.0707885104

Öhman, A., \& Mineka, S. (2001). Fears, phobias, and preparedness: Toward an evolved module of fear and fear learning. Psychological Review, 108, 483-522. https://doi.org/10.1037/0033-295X.108.3.483

Pratto, F., \& John, O. P. (1991). Automatic vigilance: The attention-grabbing power of negative social information. Journal of Personality and Social Psychology, 61(3), 380391. https://doi.org/10.1037/0022-3514.61.3.380

Pourtois, G., Schettino, A., \& Vuilleumier, P. (2013). Brain mechanisms for emotional influences on perception and attention: what is magic and what is not. Biological Psychology, 92(3), 492-512. https://doi.org/10.1016/j.biopsycho.2012.02.007

Posner, M. I. \& Rothbart, M. K. (2007). Research on attention networks as a model for the integration of psychological science. Annual Review of Psychology, 58, 1-23. https://doi.org/10.1146/annurev.psych.58.110405.085516

Qu, Z., Hillyard, S. A., Ding, Y. (2017). Perceptual learning induces persistent attentional 
capture by nonsalient shapes. Cerebral Cortex, 27(2), 1512-1523.

https://doi.org/10.1093/cercor/bhv342Robins, R. W., \& Beer, J. S. (2001). Positive

illusions about the self: Short-term benefits and long-term costs. Journal of Personality

and Social Psychology, 80(2), 340-352. https://doi.org/10.1037/0022-3514.80.2.340

Röer, J. P., Bell, R., \& Buchner, A. (2013). Self-relevance increases the irrelevant sound effect:

Attentional disruption by one's own name, Journal of Cognitive Psychology, 25, 925-931. https://doi.org/10.1080/20445911.2013.828063

Schettino, A., Loeys, T., \& Pourtois, G. (2013). No prior entry for threat-related faces: Evidence from temporal order judgments. PLoS ONE 8(4): e62296.

https://doi.org/10.1371/journal.pone.0062296

Sha, L. Z., Jiang, Y. V. (2016). Components of reward-driven attentional capture. Attention, Perception, Psychophysics, 78, 403-414 . https://doi.org/10.3758/s13414-015-1038-7

Sparks, S., Cunningham, S. J., \& Kritikos, A. (2016). Culture modulates implicit ownershipinduced self-bias in memory. Cognition, 153, 89-98. https://doi.org/j.cognition.2016.05.003

Sui, J., He, X., \& Humphreys, G. W. (2012). Perceptual effects of social salience: evidence from self-prioritization effects on perceptual matching. Journal of Experimental Psychology: Human Perception and Performance, 38, 1105-1117. https://doi.org/10.1037/a0029792

Tapper, K., Pothos, E. M. \& Lawrence, A. D. (2010). Feast your eyes: hunger and trait reward drive predict attentional bias for food cues. Emotion, 10, 949-954.

Tong, F., \&; Nakayama, K. (1999). Robust representations for faces: Evidence from visual search. Journal of Experimental Psychology: Human Perception and Performance, 25, 1016-1035. http://dx.doi.org/10.1037/0096-1523.25.4.1016 
Victeur, Q., Huguet, P., \& Silvert, L. (in press). Attention allocation to task-irrelevant fearful faces is not automatic: Experimental evidence for the conditional hypothesis of emotional selection. Cognition and Emotion.

Vogt, J., De Houwer, J., Moors, A., Van Damme, S. \& Crombez, G. (2010). The automatic orienting of attention to goal-relevant stimuli. Acta Psychologica, 134, 61-69, https://doi.org/10.1016/j.actpsy.2009.12.006

Vogt, J., De Houwer, J., Crombez, G., \& Van Damme, S. (2013). Competing for attentional priority: Temporary goals versus threats. Emotion, 13, 587-598. https://doi.org/10.1037/a0027204

Vogt, J., Bajandouh, Y., \& Alzubaidi, U. (2020). Beyond negativity: Motivational relevance as cause of attentional bias to positive information. In: H. Okon-Singer \& T. Aue (Eds.): Processing Biases in Health and Psychiatric Disorders: Neurophysiological Foundations. The Netherlands: Elsevier.

Vogt, De Houwer, J. \& Crombez, G. (2011). Multiple goal management starts with attention: Goal prioritizing affects the allocation of spatial attention to goal-relevant events. Experimental Psychology, 58, 55-61. https://doi.org/10.1027/1618-3169/a000066.

Vogt, J., Koster, E. H. W., \& De Houwer, J. (2017). Safety first: Instrumentality for reaching safety determines attention allocation under threat. Emotion, 17, 528537. https://doi.org/10.1037/emo0000251

Vromen, J. M. G., Lipp, O. V. \& Remington, R. W. (2015) The spider does not always win the fight for attention: Disengagement from threat is modulated by goal set. Cognition and Emotion, 29(7), 1185-1196. https://doi.org/10.1080/02699931.2014.969198

Vuilleumier, P., \& Huang, Y.-M. (2009). Emotional attention: Uncovering the mechanisms of affective biases in perception. Current Directions in Psychological Science, 18, 148-152. 
https://doi.org/10.1038/nn1057

Wood, N. L., \& Cowan, N. (1995). The cocktail party phenomenon revisited: Attention and memory in the classic selective listening procedure of Cherry (1953). Journal of Experimental Psychology: General, 124(3), 243-262. https://doi.org/10.1037/00963445.124.3.243

Yang, H., Wang, F., Gu, N., Gao, X. \& Zhao, G. (2013) The cognitive advantage for one's own name is not simply familiarity: An eye-tracking study. Psychonomic Bulletin and Review, 20, 1176-1180. https://doi.org/10.3758/s13423-013-0426-Z

Yantis, S. (2000). Goal-directed and stimulus-driven determinants of attentional control. Attention and Performance, 18, 73-103. https://doi.org/10.7551/mitpress/1481.001.0001

Yiend, J. (2010). The effects of emotion on attention: A review of attentional processing of emotional information. Cognition and Emotion, 24, 3- 47. https://doi.org/10.1080/02699930903205698

Yin, S., Sui, J., Chiu, Y., Chen, A., \& Egner, T. (2019). Automatic prioritization of selfreferential stimuli in working memory. Psychological Science, 30, 415-423. https://doi.org/10.1177/0956797618818483

Zhang, W., Hung, I., Jackson, J. D., Tai, T., Goh, J. O. S \& Gutchess, A. (2020). Influence of culture and age on the self-reference effect. Aging, Neuropsychology, and Cognition, 27(3), 370-384, https://doi.org/10.1080/13825585.2019.1620913

Zhu, Y., Zhang, L., Fan, J., \& Han, S. (2007). Neural basis of cultural influence on self representation. Neuroimage, 34(3), 13101316.http://dx.doi.org/10.1016/j.neuroimage.2006.08.047 